\title{
Pedagogy as Social Science and as Intellectual Helping Profession
}

\author{
Franco Blezza*
}

Professor on General and social pedagogy, Italy

ISSN: 2639-0612

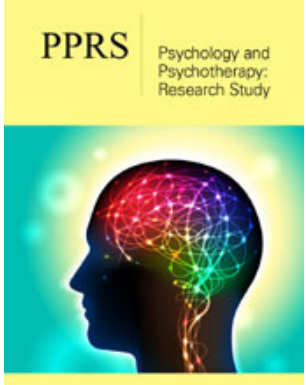

*Corresponding author: Franco Blezza, Professor on General and social pedagogy, Italy

Submission: 笽July 27, 2020

Published: 沮August 20, 2020

Volume 4 - Issue 1

How to cite this article: Franco Blezza. Pedagogy as Social Science and as Intellectual Helping Profession. Psycho Psychother Res Stud. 4(1). PPRS. 000579. 2020. DOI: 10.31031/PPRS.2020.04.000579

Copyright@ Franco Blezza, This article is distributed under the terms of the Creative Commons Attribution 4.0 International License, which permits unrestricted use and redistribution provided that the original author and source are credited.

\begin{abstract}
Pedagogy is a social science and a superior intellectual helping profession, and has 2500 years of history in both respects, significant from the very beginning. Here, we pass in a summary reviewing some historical information, the underlying epistemological issues, and a wide sample choice of procedures, conceptual and operational tools, techniques and methods of professional practice. The paper is completed by the synthetic exhibition of the pedagogical treatment of three cases of couple and parenthood problems, in which the applications of the whole is highlighted. This professional reminiscence opens the talk to future developments.
\end{abstract}

Keywords: Pedagogist; Sozialpädagogik; Professional pedagogy; Epistemology; Family and parenthood problems

\section{A Science and a Profession}

Pedagogy is a social science and a higher intellectual profession. In both respects, it has a history of over 2500 years (Dewey 1916), such as medicine and surgery or jurisprudence, dating back to the Sophists and Socrates. It is important to remember it because important components of its hinterland, and many of its conceptual and operational tools, date back to those origins, as we will mention. It cannot be limited to a theoretical (or "theoretical") study of education, it is not a "logy". There has been an attempt to found a "pedology" (paedology/ paidology/paedology) by Oskar Chrisman (1855-1920) in the book Paidologie. Design to a child's science (Druck von Bernhard Vopelius, Jena 1896), but in this sense it has proved sterile.

Someone, with little epistemological knowledge and even less scientific culture, speaks about a "practical science", but it is a gross error. Science is pure knowledge, whose products are formulas, laws, theories and the like. If anything, there is technique, the reflection on which it is called "technology", but thereby are other things. In this case too, Durkheim's importance as a pedagogist is clear to us.

It goes not without essential meanings that pedagogy is the only science and the only profession that has the suffix "agogy", which recalls a direct relationship with the reality of education, sociability and being educated peoples, and implies taking care of it as shown in Martin Luther King's well-known statement “I care!". There are also andragogy and geragogy, but they indicate branches of pedagogy having the name with the same suffix propagated, and in addition to everything in western languages, the comprehensive term "pedagogy" prevails, even for ages after the development ones. Pedagogy, in summary, is a field of engagement to education by reflection and application whose profession practice on an intermediate dimension between theory and practice, the applicative plan, in German possible application and, with certain authors, Empirie. It is one among the professions by the word. The role of the professional pedagogist is defined by practicing a specific form of helping relationship/ counseling, upon request, in the presence of problematic situations of educational, social, and relational nature. This professional practice is a special form of let's talk/alone, parlons!/ Come on, let's talk!/Su, parliamo. It's named "pedagogical interlocution". In the context of this 
professional practice we could also implement our conceptual and operational tools as well as methodological, procedural, and lexical resources [1,2]. The aim of this paper is to outline the general characteristics of the professional approach of the pedagogist and summarize some of the various forms of the complex tools that can be used in this practice from the original ancient Greek and Latin work to the recent reports Orefice et al. [3,4]. Finally, two examples of professionally treated cases are discussed in the necessary summary. There will be no conclusions to be drawn, as scientific research continues in the context of this professional practice.

\section{Basic Fonts and Tools}

Perhaps, some resemblances or similarities to other professions by the word ("let's talk!") can be detected; but there are fundamental differences that emerge immediately, particularly, the non-therapeutic character of the professional pedagogical practice, the exclusion of any digging into the unconscious and the work on transferable inter-personal experiences and evidences instead of Erlebnis (life experience/lived experience, but the technical term is international and should not be translated). It is a helping relationship about consciousness, which may have long been a conscience left unintended, unconfirmed, taken for granted, or not necessarily the object of any special attention. The role of the pedagogist does not involve finding solutions: it is rather related to helping the interlocutor to find his own way to solve the problems; or, better, to try a solution, to hypothesize it, since in pedagogy, as in every science stricto sensu, we do not have any truth criterion. The pedagogist follows the so-called clinical method [5,6], i.e. casuistricsituational method, the reciprocal of the statistical-operational method. This methodological choice implies approaching the social and relational context, in which the interlocutor is located, and must get into the problematic situation of the interviewee and reelaborate it in order to make the situation more easily assumable and resoluble.

We could sketch the stages of pedagogical interlocution as follows (Blezza, Crispiani).

a. Identifying the problematic situations and helping the interlocutor to intentionally undergo a transition towards full problem posing by reacting positively and constructively to the situations.

b. Soliciting and promoting the practice of the faculty of creativity and recalling the experience of proposing hypotheses in solving the posed problems. This practice is always hypothetical and fallible, and hence it allows the process of screening the best solution for the interlocutor himself.

c. Respecting the rules of "internal" coherence, that is the logical one, with the removal of the contradictions that emerge in the discussion on the various hypotheses introduced in the social and cultural context of the interlocutor.

d. Respecting the rules of "external" coherence, that is the empirical or factual one, with the examination of the advanced hypotheses, as far as possible, generating a virtual criticism of the whole cultural context. This analysis aims to discard the hypotheses that are falsified by the experience that follows (the "future experience" of Pragmatists) and to maintain the ones that constitute a provisional force susceptible to subsequent falsifications.

e. Sound opening to the evolution of ideas and self, the benefit of the doubt, and the critical spirit.

f. And always and still the ideas for man, vice versa not being pedagogically admissible.

\section{The life project(s)}

Based on the aforementioned stages, we can state the fundamental tools of today's pedagogical interlocution as follows: identifying and solving problems, the practice of human creativity, internal and external coherence, and all that is the professional exercise of pedagogical counseling. In this paragraph we will summarize a fundamental example of recent additional tool currently employed by pedagogists [1]. The pedagogical interlocution is related, first of all, to the "life project" which concerns every human being and the people surrounding him. This project is not a plan or model, and therefore it is always susceptible to reviewing, rethinking, and correcting. The life project often poses problems regarding internal or external coherence previously discussed, because sometimes it can be unrealistic or intrinsically contradictory. In social relationships, there are often logical or empirical contradictions as far as a person's project concerns the future of related people such as the partner, the children, the relatives, the work colleagues, and so on: generally speaking, the social network. To exemplify, we will discuss a particular case of a couple where each partner "naturally" assumed at the time the other one's own choices and renounces definitively without a proper discussion. The pedagogist uses "word and reasoning" in this case inviting the interlocutors to discuss and compare their respective life projects explicitly with all the underlying details regarding the personal character of the other partner.

\section{Tools of Greek (and Romsn. Latin) origin}

The classical Greek culture is the first source of tools for pedagogy. We can bear witness to its wealth and importance,

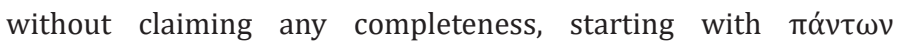

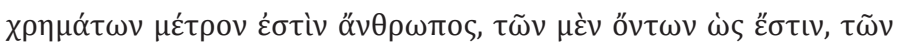

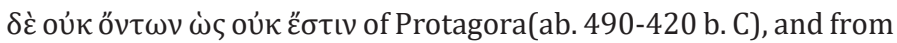

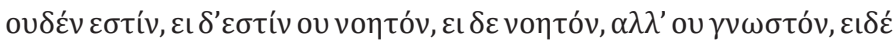
$\kappa \alpha \iota \gamma v \omega \sigma \tau o ́ v, \alpha \lambda \lambda^{\prime}$ ou $\delta \eta \lambda \omega \tau$ tóv $\alpha \lambda \lambda$ ous of Gorgia (ab. 485-380 b. C) and active participation in their own reality or with the Aristotelian

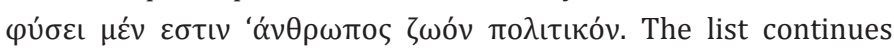
with logic, from before Aristotle to the latest developments: with the $\gamma \vee \sigma \theta \imath \quad \sigma \varepsilon \alpha v \tau$ ó $v$ and the deep sense of human limitation and the resulting condemnation of the $\vartheta \boldsymbol{\beta} \rho \varsigma 1$ 'superb and arrogant violation of this character, tools and warning of the latter's evident momentum. Much of the Greek philosophy, including the described examples, can be summarized on the basis of the colossal scientific culture of the people: knowing that it aroused our admiration, but that it was never intended for the application, 
even though it included electricity, magnetism, heat transformation in mechanical energy, and many other knowledge that would revolutionize human life in different periods, with particular regard

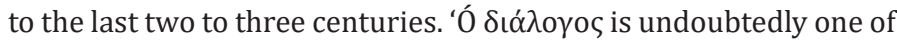
the most important tools for the entire professional career of the pedagogist. In his Socratic form reported by Plato, the founder of other professional exercises can be identified, not excluding the same psychoanalysis, even in his more classical formulation.

Remembering that it was not born with Socrates, for example it was widely present in the tragedy, and has had important developments and applications in different and later epochs and cultural realities, it is surely one of the fundamental tools for us today, but with a substantial change. While we can substantially accept the conception of $\eta \dot{\varepsilon} \varepsilon \omega v \varepsilon \varepsilon^{\prime} \alpha$ according to Socrates, we will

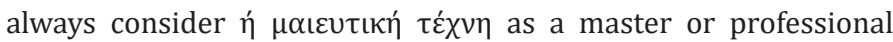
aid to the pupil and the interlocutor to shed light on how much he can develop within himself, but for this reason, we would never use $\eta^{\prime} \alpha \lambda \eta \dot{\theta} \theta \varepsilon \iota \alpha$ concept. The truth, in any case. While there is falsehood and is also called into question with irony, no product of human creativity can attribute the character of truth, except in an interlocutory, provisional, hypothetical sense, which can never be released from doubt and by the critic, and who is always susceptible to subsequent falsifications, for how many empirical confirmations he has received. Having said that, it would not be difficult to entertain ourselves also on the professional tools of the pedagogist of Roman and Latin origin, starting from Cato censor, Seneca, Lucretius, Quintilianus, and considering also that the Romans were masters of law and of technique as the Greeks were masters of science. But the economy of the paper requires us to redirect this discourse to the long history of pedagogy, taking it up again in recent centuries.

\section{Institutional and school pedagogy, and professional pedagogy}

Any discourse can be made about the functional use, and sometimes the essential use, of professional pedagogy, and more generally of social pedagogy, of tools developed in the field of school and institutional pedagogy. This is not just a matter of starting from Ickelsamer and Comenius (XVI century), recalling the last few centuries: this has already been done to an appreciable extent, and of course it is susceptible to further substantial evolution. We should start at least from the educational and didactic visions of the middle ages such as the teaching of Latin, with a powerful focus on the formalized aprioristic rules of grammar and syntax. These rules also extend to the Greek after humanism, which still hinder the mastery of the four skills that come from "modern" languages in a few years, and in fact prevent the start of classical language study at the university for those who do not have the substantial previous details some high schools address. But we cannot go beyond these brief notes and the simple enunciation of further examples: the active and passive curriculum, the historically remote concept of the program and its integration with the programming concept, and the three phases of the evaluation have a remote history and their respective different functions for the learner as well as the teacher; and so on

\section{The foundation of the Sozialpädagogik}

If the pedagogical professional practice dates back to the Sophists, its next recent ground is attributed to the foundation of the Sozialpädagogik, in the Mitteleuropa of the XIX century. This foundation in that sociocultural context, which also gave rise to other sciences, demonstrated to be a cornerstone for professions for the following century: Karl Mager, Friedrich AW Diesterweg and the transposition of Pestalozzi's pedagogy, Paul Natorp [6], Émile Durkheim in his primary contribution as a pedagogist. Here it is necessary to look for the roots of a social profession, and not indulge in simple social declarations of pedagogical discourse. Society as such is the domain of general pedagogy-allgemeine Pädagogik: the definition of a branch, like in any other science, involves the rigorous identification of a particular domain and other peculiarities. It is significant that scientific psychology originated in the same temporal and cultural context, as did psychoanalysis a few decades later; the centrality of the word and the senses in these and other similar sciences allow the identification of a synergy that is not easy to implement, but has an absolute evidence.

\section{Psychologie, psychoanalysis and "sciences of education"}

In the second half of the nineteenth century, in Mitteleuropa or on its borders, not only the Sozialpädagogik was born: were scientifically founded several social, man and culture sciences that gave rise to professions that became established in the following century, such as scientific psychology, sociology, anthropology and so on: generally speaking, die Human- Social- Geistes-Wirtschaftswissenschaften. These sciences offer also well understandable tools for professional pedagogy and the pedagogist. We call them "sciences of education" in the sense that they are sciences that offer great contributions to education and the related professions, with the pedagogy that reprocesses and turns them to the aims of education, which are not relevant purposes proper to these sciences [7-9].

It is necessary to point out and to repeat, for so many meaningless speeches that have been heard in this specific regard, that we speak of stricto sensu sciences, both about pedagogy and about the sciences of education, according to the Popper [10] "Abgrenzungskriterium ", i.e. "An empirical business system can be used as a means of production". It's a statement relevant for the psychological and medico-surgical sciences, too. Among the sciences of the XIX century which became social professions in the $\mathrm{XX}$ century, the last and most noteworthy was the psychoanalysis of Freud S [11]. The pedagogist is not a psychoanalyst, and not only because he does not delve into the unconscious, nor does he have to approach this profession; but it must have an adequate psychoanalytic culture.

Moreover, many are the conceptual tools proposed by the Frankfurt philosopher, psychoanalyst and sociologist Erich Fromm [12], and we have experienced; in particular, "paternal" or conditioned love and "maternal" or unconditional love, whose balanced synthesis is the fullness of love that can be considered an art (1963); the concepts of biophilia and necrophilia also to study the problems of violence $(1975,1983)$; the social vision of the state 
of health and illness (1996). Particularly, Fromm's lines of action cover the psychoanalytic and therapeutic side of some fundamental issues, leaving free and evident emphasis in those same cases the field of pedagogical intervention towards educational imbalances and deficiencies. Also, there are just two examples, albeit of enormous importance, of scientists of the last centuries who offer essential contributions of educational sciences to the pedagogist's toolbox and practice. We can conclude this paper with some synthetic examples of cases professionally treated, thus respecting the scientific methodology and the nature of pedagogy, so that the organic relationship with the experience is indispensable. Some of the instruments outlined above have been applied [1316]. This is our application of the Abgrenzungskriterium, this is the way we respect the "future experience" following James and the pragmatists, the way of "external coherence". Ample clinical cases and exemplifications are contained in some works in the bibliography [1].

\section{The case of a couple to balance}

We will begin here with the reminiscence of one of the very first cases that we treated professionally, to be precise in the year 1990. This case concerned a couple of 41 years old and 45 years old, married since 1970 with two sons of 18 and 3 years old and a daughter of 16 . We pay attention to the fact that their relationship, an engagement of many years before the wedding, had fallen into the very last days of the Bürgergeist nineteenth-twenty century; in particular, "certain things" should not be mentioned. The couple, indeed, had met at the end of the 1950s, when the nuclear family was still paradigmatic undiscussed. Considering their lives and the different sexes, the couple had attended different high schools after which the husband had a diploma (he was graduated from a high school). Then he attempted ambitious university studies by not combining anything, and in the end giving up and falling back on a job corresponding to the diploma. The wife, on the other hand, had to abide by the conventions of that era where the woman should not hold higher degrees than her husband. Although she was passionate about her university studies, she had to quit university after she had successfully completed half of the requirements of her chosen major. In those days, graduating in Italy took four or five years, six years only for medicine and surgery. The marriage followed shortly after the double renunciation of university studies, and the couple lived together for many years a settled life. Shortly after the second "happy event", the birth of the second child, the daughter, some symptoms had appeared on the wife such as loss of control of her actions and hysterical behaviors (this is their common speaking; the diagnosis must be made by the doctors). These crises had soon led to the wife often refusing (not ever!) her husband and searching for adventures [17-21].

About how the third conception occurred in contrasting couplings, each of the two gave several strongly contradictory versions; however, the birth of the third son did not improve things and precipitated them; if at that time the genetic research of paternity was available, they were not aware of it. The two partners approached the pedagogist with considerable delay, one might say: but this only happened when they, first of all, learned that they could have a form of help other than a medical, psychiatric or psychotherapeutic treatment that both had excluded, starting with the man. Surprisingly, the spouses were immediately and easily open to discuss their intimate life together although such conversations were considered as taboos in their time, we observed. The times were changing. Their stories were absolutely converging, without contradictions [22-26]. The "bourgeois" origin of the husband defined his social and sexual behaviors. He was formed by an education where the husband was the consumer and the wife was the one who sacrificed to recharge him. He said "I was convinced that she was not interested in sex, and that she wished to get rid of this task in the quickest and least expensive way possible"; instead she remembered, "only the first two times I experienced something, precisely a strong pain; then I felt nothing more and wondered if sex was this poor thing". Their sexual life was characterized by repetitions with lack of imagination and preliminary actions. Silent and quick. The couple had no difficulty in identifying the major source of dissatisfaction related to the sacrifices of the wife, especially regardless of her needs. Despite that the couple had never discussed their problems before, their openness to all questions and willingness to change their behaviors led them to rediscover the strong emotional and social bond between them. They commenced to be more imaginative in their sexual relationship, focusing more on the partner's need of affection and satisfaction. Four meetings with the pedagogist were sufficient, in addition to the preliminary one and a further one month later. The couple succeeded in rediscovering one another and to revive the marriage. They redecorated their bedroom reuniting the previously separate beds and they managed to remain united as family for decades after. He died in a car accident recently, until then the couple was absolutely united. I know that the daughter had some problems, for which, however, they turned to a psychotherapist, and I ignore every detail.

\section{The case of a neo-nuclear family}

As well known, the nineteenth to twentieth century era was characterized by a frenetic social, cultural, economic, productive, transport, communications evolution and so on. The necessary condition for keeping that socio-cultural reality and the relative heavy sacrifices, and cell of that society, was the family built strictly corresponding to the "nuclear" or "conjugal" paradigm. This conventional family consisted of a couple with rigidly fixed roles. The genres were polarized by the specific education to the extreme as a result of a powerful educational system with oppressive and violent determination. The role of the male was the investment outside the house such as housing, constructing, and maintaining the home hearth, and controlling the whole family social relationships. On the other hand, the female had the complementary "inside" roles. This quite unnatural paradigm was not the mere product of ancient civilizations or traditions; it was the answer to the bourgeois revolutions of the late XVIII century. This family structure is now suffering from irreversible crisis for decades, and the end of this crisis is not visible after more than half a century, even if in professional pedagogy we have alternative proposals of some interest. 
It is worth noting that "neo-nuclear" families can exist nowadays and can work in an excellent way. The difference is placing the two partners at the extremes of that polarization as the result of a free and common choice and not an educational duress like the one in the past. In addition, it is not a must anymore that the male is the one who invests totally "out". Indeed, the exchange of roles is becoming more and more frequent. In our experience, it is the most frequent case. One of these cases is related to a couple of spouses with a daughter possessing a firm (a fashion trade activity) where the expectation of success was entirely relied in the woman. She was the only daughter engaged in that parent's business, and the typology with expected huge profit: a profit that came only to a small extent. Both the daughter and her husband were convinced that the parents' inability and shortcomings were the reasons for their deficit. The younger couple in this family was originally peers who came together at the age of 15-16 year. The daughter was working in the firm since her childhood and the parents payed her contributions for retirement since her 14th birthday, according to Italian law. She later obtained a diploma (was a high school graduate) with considerable difficulties. The son-in law completed his studies up to his university degree in a highly professional sector. Nevertheless, he had opted for a modest, fixed-term and near-home job, also in order to be able to follow their daughter and the house. He was married about 3 years after his graduation. The foreseen arrangement was shortly changed after one of his wife's parents died and the other one chose the retirement, surrendering the firm to the daughter. Despite that, their standard of living remained modest for about twenty years. It is important to note that the young couple placed great importance on status symbols for themselves and paid a deal of attention to their appearance, and hence their low budget could raise some questions. The younger family is coherent with the nuclear paradigm where the husband abstains from collaborating with his wife in spite of the convenient environment for his collaboration that would have been yielded a valuable outcome, as testified by our collected data. When the wife and dealer turned 49 , she had already spent 35 years of contributions which was the approved time of legislation for retirement from of the work. She took the resolution of taking care of her mother. The sale of the firm covered their relatively huge debts. At that time, the son-in law faced a very serious crisis: he began to quarrel, scream at home, quarrel with his wife, and to refuse her.

Hence, the couple decided to consult the pedagogist for help. It was not difficult for the couple to identify the solid contradictions in their life projects that were never minimally discussed explicitly. He remembered in details all his career prospects he had declined after graduation as renunciation to support her business, expecting luxury and glamor as the definite outcome. In her turn, the daughter explained that she had tried to correct what she had identified as parents' mistakes in running the company, but it was not enough to save the firm from quite low levels of survival. She also mentioned that her commitment started when she had missed her chances of studying and living as a student. The daughter declared that she never asked her husband for any sacrifice, and that he had the expectation of a better chance of living by marrying a graduate of a good professional sector. The couple had finally spoken extrovertly with fundamental intellectual honesty. Nevertheless, none of them showed any willingness to change. Hence, no one had presented the essential condition for pedagogic treatment in this case that can be described as "opening", or "openness". The couple insisted on their ideas: the wife demanding her husband to follow alternative career path, the husband replying that it is impossible at the age of 50 and accusing his wife of inadequacy in developing her business in the interest of the their family and daughter. This relationship was ended by a violent and dramatic separation after rapid deterioration followed by the husband's new affair with another woman. We don't know about her. The case could be treated pedagogically if the two partners had demonstrated the necessary openness, and above all if they had turned to the pedagogist in time. At that point, it was no longer a matter for a pedagogist but for other legal professionals.

\section{The derivative case of a dazed daughter}

It is important to state the couple in this case was not the one who referred to the pedagogist. The professional pedagogical help had not solved the case. But there was still something to do, some of the utmost importance. Their daughter, now a University student registered in another nearby city, she had been shocked and confused by her parents' quarrels, and had stopped attending and studying: remember that in Italy there are no time limits for obtaining a university degree. She called the pedagogist, had sought this help after being criticized by her parents as desperate and out of perspective. The granddaughter in this case who sought help had kept some illusions about the fate of her parents' relationship. In reality, they have given themselves unfounded projects and have not been able to act correctly or correcting the numerous errors that they made. The possibility here is to retrieve the life project of this girl without carrying the heavy loads of a father recovering his personal image and a self-employed mother who can be ultimately a social subject. What her parents were like was really a misery and it would not be enough to continue the studies, but she could have received the necessaries from her grandparents, alive, or hereditary. The case, which was not a positive solution to the parents, was resolved without difficulty in the daughter. The girl had resumed regular attendance at the university, with full personal satisfaction as if it were a happy rediscovery and overtaking quite early some relatively demanding examinations. The image of herself that this girl had to help herself to build was not that of a trader's daughter, but her own of a student passionate about the subject she had chosen. A first contact, three meetings and a further recall meeting had been enough to restore the student to the society, and the selfimage and Lebenssinn to the girl.

\section{References}

1. Blezza F (2018) Professional pedagogy. Libreria Universitaria, Limena, Italy.

2. Crispiani P (2001) Pedagogia clinica. Azzano San Paolo, BG, Italy.

3. Orefice P, Carullo A, Calaprice S (2011) The educational and training professions: From the social question to the legislative response, CEDAM, Padua, Italy.

4. Orefice P, Corbi E (2017) The professions of educator, pedagogist and researcher pedagogist in the European framework, ETS, Pisa, Italy. 
5. Massa R (1992) The training clinic. F Angeli, Milano, Italy.

6. Blezza F (2010) Social pedagogy. In: Liguori, Napoli, (Eds.), Italy.

7. Mialaret G (1976) Educational Sciences, Puf, Paris, France.

8. Visalberghi (1978) Pedagogy and Educational Sciences, Mondadori, Milan, Italy.

9. Dewey J (1929) The sources of a science of education. Horace Liveright, New York, USA.

10. Popper KR (1935) Logic of research-on the epistemology of modern natural sciences. Springer-Verlag, Wien, Germany.

11. Freud S (1940-1952) Collected works. Ordered chronologically. 17 volumes, plus a register volume (volume 18) and a volume with supplements (volume 19). In: Anna Freud, First published by Imago publishing co., London, UK.

12. Fromm ES (1973) The anatomy of human destructiveness. Holt, Rinehart and Winston, New York, USA.

13. Borrelli M, Ruhloff J (1998) German contemporary education. Schneider Verlag Hohengeren, Baltsmannweiler, Germany, Volume (1-3).

14. Catarsi E (2008) Pedagogy of the family. Carocci Publisher, Rome, Italy.

15. Catarsi E, Pourtois JP (2011) Family education and services for childrenfamily education and services for children. Florence University Press, Florence, Italy.
16. Crispiani PC (2008) Giaconi autoanalisi. Azzano San Paolo, BG, Italy.

17. Crispiani P (2015) Giaconi Diogene 2016. Azzano San Paolo BG, Italy.

18. Dewey J (1916) Democracy and education. The Macmillan Company, New York, USA.

19. Durkheim DE (1938) Pedagogical evolution in France (Course for candidates for the aggregation dispensén (Paris, 1904-1905), Libraire Félix, Paris, France.

20. Durkheim DÉ (1968) Éducation et sociologie (1921). Les Presses universitaires de France. Paris, France.

21. Formenti L (2004) Pedagogia della famiglia. Guerini, Milano, Italy.

22. Fromm ES (1956) The art of loving. Harper \& Row, NewYork, USA.

23. Fromm ES (1983) About love for life. Deutsche Verlags-Anstalt, Stuttgart, Germany.

24. Fromm ES (1991) The pathology of normality. In: Iori V (Ed.), To the science of man. Quadriga, Berlin, Germany.

25. Rezzara (2004) From pedagogical science to training clinic. F Angeli, Milan, Italy.

26. Telleri F (2006) Pedagogical consultancy and mediation, Carlo Delfino publisher, Sassari, Italy. 